\title{
Meta-analysis of current chemotherapy regimens in advanced pancreatic cancer to prolong survival and reduce treatment-associated toxicities
}

\author{
JIE CHEN $^{1,2^{*}}$, LINLI CHEN ${ }^{3 *}$, JIANPING YU ${ }^{4 *}$, YANMEI XU ${ }^{3 *}$, XIAOHUI WANG ${ }^{5}$, \\ ZIQIAN ZENG ${ }^{6}$, NING LIU $^{7}$, FAN XU ${ }^{6,8}$ and SHU YANG ${ }^{6}$
}

${ }^{1}$ School of Chinese Medicine, Li Ka Shing Faculty of Medicine, The University of Hong Kong, Hong Kong, SAR 999077; ${ }^{2}$ Department of Orthopedics, Shanghai Institute of Traumatology and Orthopaedics, Ruijin Hospital, Shanghai Jiaotong University School of Medicine, Shanghai 200025; ${ }^{3}$ Division of General Practice,

West China Hospital, Sichuan University, Chengdu, Sichuan 610041; ${ }^{4}$ Department of Neurology,

The First Affiliated Hospital of Chengdu Medical College, Chengdu, Sichuan 610500; ${ }^{5}$ Department of General Surgery,

Bayingol Mongolia Autonomous Prefecture People's Hospital, Urumqi, Xinjiang Uygur Autonomous Region 841300;

${ }^{6}$ Public Health School, Chengdu Medical College, Chengdu, Sichuan 610500; ${ }^{7}$ Department of Medicine,

Sunshine Guojian Pharmaceutical Co., Ltd., Shanghai 201203; ${ }^{8}$ Interdisciplinary Division of Biomedical Engineering,

The Hong Kong Polytechnic University, Hong Kong, SAR 999077, P.R. China

Received April 2, 2018; Accepted October 23, 2018

DOI: $10.3892 / \mathrm{mmr} .2018 .9638$

\begin{abstract}
Unresectable advanced pancreatic cancer (APC) is a highly lethal malignancy. Although numerous chemotherapeutic regimens are available, evidence regarding the survival extension, the life quality improvement, the associated risks and occurrence rates of adverse effects, is required. The effects of 19 chemotherapy regimens on survival and treatment-associated toxicities in the context of APC treatment were comparatively assessed. A total of 23 randomized controlled trials were included in this network meta-analysis. For overall survival, five regimens, Gemcitabine (Gem)+radiotherapy (Radio), Gem+cisplatin (Cis), Gem+erlotinib (Erl)+bevacizumab (Bev), Gem+capecitabine (Cap)+Erl, and Gem+exatecan, were the most effective treatments, according to their respective high surface under the cumulative ranking (SUCRA) probabilities. Regarding the progression-free survival, five
\end{abstract}

Correspondence to: Professor Fan Xu, Interdisciplinary Division of Biomedical Engineering, The Hong Kong Polytechnic University, Block S104, 11 Yuk Choi Road, Hung Hom, Kowloon, Hong Kong, SAR 999077, P.R. China

E-mail: xufan@cmc.edu.cn

Dr Shu Yang, Public Health School, Chengdu Medical College, 783 Xindu Avenue, Chengdu, Sichuan 610500, P.R. China

E-mail: shuyangcmc@163.com

*Contributed equally

Key words: unresectable advanced pancreatic cancer, chemotherapy regimens, benefits and risks regimens, including Gem+Radio, Gem+Erl+Bev, Gem+Cis, $\mathrm{Gem}+\mathrm{Cap}+\mathrm{Erl}$ and Gem+pemetrexed, were the most effective treatments based on their SUCRA probabilities. Each regimen exhibited advantages and disadvantages, and 14 common treatment-associated toxicities were present in different proportions. The three principal toxic effects included haematological, gastrointestinal and constitutional symptoms. To improve survival, chemotherapy regimens with high SUCRA probabilities require prioritizing. Although treatment-associated toxicities are unavoidable, the regimens presented toxicities in distinct proportions. Therefore, clinicians should assess the disease status of the patients, and balance the benefits and risks of the selected treatment.

\section{Introduction}

Unresectable advanced pancreatic cancer (APC) is the most lethal and the most aggressive human cancer (1). APC is predicted to increase from the 4 th to the 2 nd leading cause of mortality in the USA by 2020 due to its lethal and malignant characteristics (2). Due to the limitations of diagnostic techniques, the majority of patients and clinicians become aware of the disease too late, as this cancer is frequently diagnosed in an advanced stage $(3,4)$. APC is characterized by a high mortality rate worldwide, $90.8 \%$ in China (5), $78.5 \%$ in the USA (6) and 95.0\% in Canada (7).

Gemcitabine (Gem) was more effective compared with 5-fluorouracil (5-FU) in patients with APC and improved the survival rate; therefore, it was approved as a first-line regimen by the US Food and Drug Administration (FDA) in 1996 (8). At present, the majority of chemotherapy regimens are derived from Gem, which was used as the control treatment in numerous previous studies (9-11). 
Although a number of combination chemotherapy regimens containing Gem $(\mathrm{Gem}+\mathrm{Xs})$ or monotherapies have become more prevalent over the past decades (12), the improvement of the conditions of the patients has been limited (12-15). For example, the poor prognosis of APC leads to a low survival rate $(14,16,17)$, which has remained relatively unaltered for $\sim 5$ decades (15). Nevertheless, the benefits and risks of combination chemotherapy regimens remain unclear. Therefore, first-line chemotherapy regimen data were pooled to comprehensively evaluate the benefits and risks of these treatments.

\section{Materials and methods}

Study design. In order to assess the benefits and risks of various chemotherapy regimens in distinct conditions, head-to-head comparison clinical trials were selected. This network meta-analysis followed the preferred reporting items of system reviews and meta-analysis (PRISMA) statement (18) while integrating evidence from direct and indirect treatment comparisons (19). The flow chart for study selection is presented in Fig. 1.

Search strategy. The comprehensive search strategy was conducted using the MEDLINE (www.pubmed.com), EMBASE (www.embase.com), Cochrane Central Register of Controlled Trails (www.cochranelibrary.com) and ClinicalTrials.gov (https://ClinicalTrials.gov) databases with the following search terms: (Advanced pancreatic cancer or pancreatic cancer) AND (advanced pancreatic cancer or chemotherapy regimens). The drug abbreviations and the combinations tested are listed in Table I.

Study selection criteria and outcomes. Experienced investigators independently selected the studies and extracted the data, and any conflicts were resolved in discussion. The study selection criteria were based on the National Comprehensive Cancer Network 2017 criteria (20). The following inclusion criteria were applied: (i) Parallel-group randomized controlled trials (RCTs; phase II or III) with the Gem intervention set as the common comparison treatment and including $\geq 2$ arms; (ii) a minimum 6-month follow-up period; (iii) patients $\geq 18$ years old (i.e., adult patients); (iv) diagnosis of unresectable APC; (v) application of palliative treatments, including invasive radiation therapy, chemotherapy or chemoradiation therapy, targeted therapies or combination therapy with the respective placebo or control group; (vi) either fixed-dose or flexible-dose RCTs with dose titration; and (vii) the patient performance status reported as 0-2 scores in the Eastern cooperative oncology group or 70-80\% in Karnofsky scales (21). Previous studies that (i) included patients undergoing radical resection, (ii) failed to report the number of patients, (iii) failed to report the primary efficacy outcome [progression-free survival (PFS)], or (iv) failed to report the data necessary to estimate the standard deviation of the primary efficacy outcome were excluded.

Data extraction. The following data were extracted from each included RCT: First author's name, published year, clinical phase, sample size of each arm, age, treatment, dosage, route, duration, overall survival (OS) in months, PFS in months, and 14 treatment-associated categories of side effects
Table I. Abbreviation list of chemistry regimens.

\begin{tabular}{ll}
\hline Abbreviation & \multicolumn{1}{c}{ Chemistry regimens } \\
\hline Gem & Gemcitabine \\
Gem+Axit & Gemcitabine+axitinib \\
Gem+5-FU & Gemcitabine+5-fluorouracil \\
Gem+Cap+Erl & Gemcitabine+capecitabin+erlotinib \\
Gem+Cap & Gemcitabine+capecitabine \\
Gem+Cet & Gemcitabine+cetuximab \\
Gem+Cis & Gemcitabine+cisplatin \\
Gem+Erl & Gemcitabine+erlotinib \\
Gem+Erl+Bev & Gemcitabine+erlotinib+bevacizumab \\
Gem+Eta & Gemcitabine+etanercept \\
Gem+Exa & Gemcitabine+exatecan \\
Gem+Iri & Gemcitabine+irinotecan \\
Gem+Mar & Gemcitabine+marismastat \\
Gem+Nab-p & Gemcitabine+nab-paclitaxel \\
Gem+Pem & Gemcitabine+pemetrexed \\
Gem+Radio & Gemcitabine+radiotherapy \\
Gem+Sor & Gemcitabine+sorafenib \\
Gem+Tip & Gemcitabine+tipifarnib \\
Gem+Vis & Gemcitabine+vismodegib \\
Oxa+Iri+Leu+Flu+Inf & Oxaliplatin+irinotecan+ \\
& leucovorin+fluorouracil+infusion \\
\hline &
\end{tabular}

associated with quality of life ('hepatotoxicity', 'haematological', 'mental/psychiatry', 'renal toxicity', 'gastrointestinal', 'neuropathy', 'electrolytes imbalance', 'pain', 'infection', 'skin', 'constitutional symptoms', 'cardiac/vascular', 'pulmonary' and 'other'). The details of the outcomes of the included studies are presented in Table II.

Risk of bias assessment. To reduce the risk bias, the recommended approach of Cochrane reviews was followed and the risk was assessed throughout the process (22). The following bias sources were independently assessed: Random sequence generation, allocation concealment, blinding of investigators and/or patients, blinding of outcome assessment and the degree of data incompleteness. Each bias was scored as low, unclear or high, as presented in Table III.

Statistical analysis. All statistical analyses were performed using the network meta-analysis package in Stata (version 13.0; StataCorp LP, College Station, TX, USA) (19). For the endpoint outcomes, OS and PFS data were extracted from references as medians and subsequently transformed into standardized mean differences with $95 \%$ confidence intervals (CIs). A network meta-analysis was conducted following the standard workflow (19). The network map presents the connection status of the studies; no loops and a P-value $>0.05$ validated the consistency model to perform the network meta-analysis. Heterogeneity was assessed with the $\mathrm{I}^{2}$ metric. Heterogeneity was $0 \%$ for OS and 75.64\% for PFS. Therefore, the Mantel-Haenszel fixed-effects model was used for OS, and the Mantel-Haenszel random-effects model was used for PFS (21). All of the studies with various 


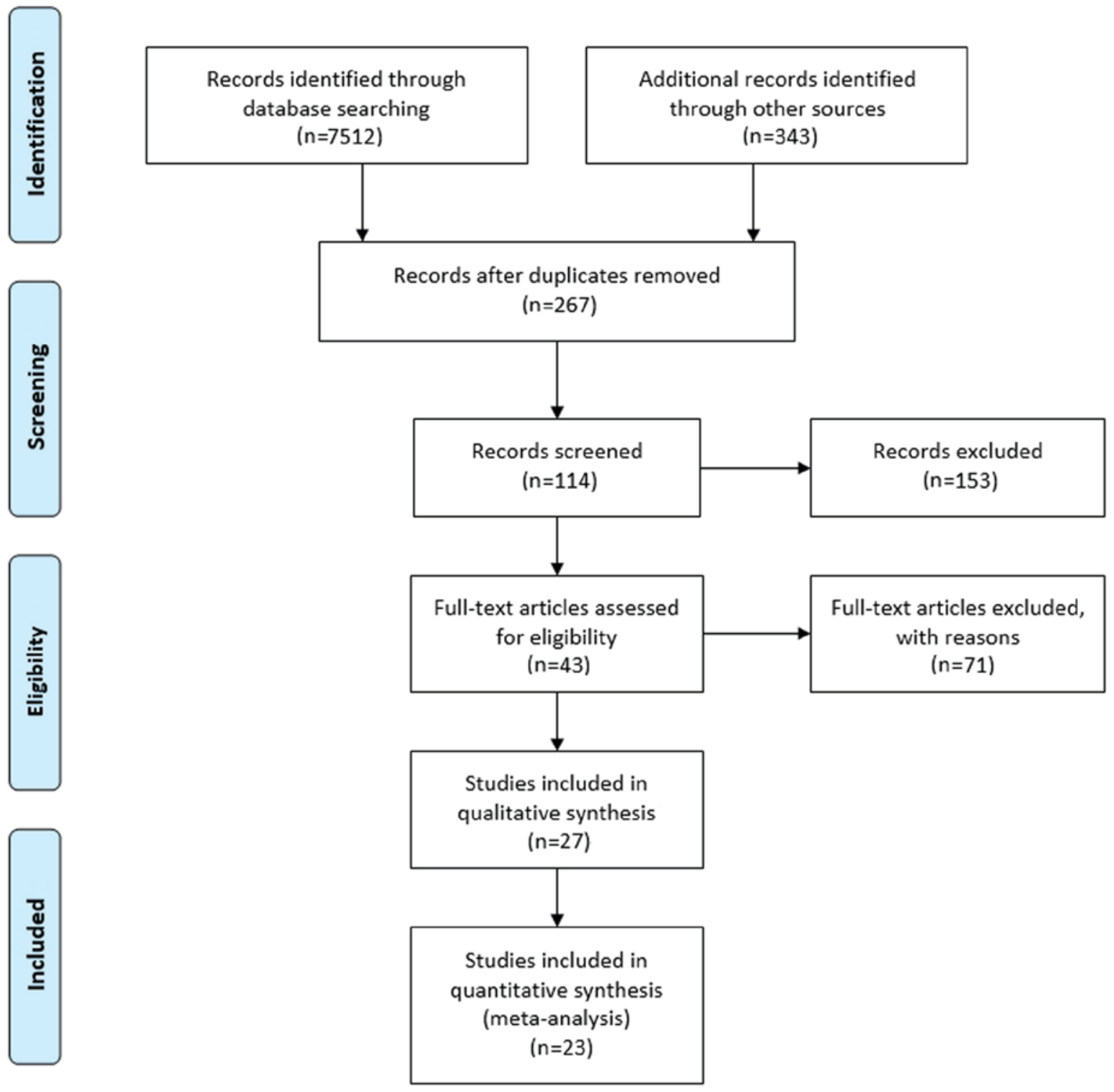

Figure 1. Flow chart of study selection.

treatments were included as drugs that were either directly or indirectly associated with a common comparator (Gem only) for further downstream ranking analysis. Subsequently, to rank the effects of the treatments, the analysis of the surface under the cumulative ranking (SUCRA) probabilities was performed under the protocol of Stata (19), and the results are presented as the percentage of the efficacy of each intervention relative to a hypothetical ideal intervention (23). A larger SUCRA score indicated longer OS and PFS.

The data are presented in an ordinal data format according to the 14 categories of side effects. Network meta-analyses were separately conducted, and the data were calculated as hazard ratios with $95 \%$ CIs. To examine and classify the adverse effects that occurred among the different treatments, a stack bar graph of each category was generated.

\section{Results}

Standard workflow via PRISMA. To ensure the general quality of the present study, a PRISMA flowchart regarding the screening process of the study used, is presented in Fig. 1. From an initial set of 7,855 non-duplicated studies, a total of 23 RCTs were included in this analysis. The drug abbreviations and the combinations assessed are listed in Table I, and the general characteristics of the included RCTs or studies are presented in Table II. The risk of bias assessment for these included RCTs is depicted in Table III. The geometry evidence of the OS network plot and its associated pooled forest plot are summarized in Fig. 2. Furthermore, the geometry evidence of the PFS network plot and its associated pooled forest plot are summarized in Fig. 3. Using SUCRA, graphs of the rank of the treatments associated with OS and PFS are listed in Fig. 4, and the 14 types of treatment-associated toxicities are presented in Fig. 5.

Network diagram (geometry and forest). Numerous combinations of various treatments were analyzed. The network maps of OS and PFS demonstrated the geometry of 18 chemotherapy regimens compared with a common treatment, Gem, and noloops were identified (Figs. 2A and 3A). Furthermore, the network 


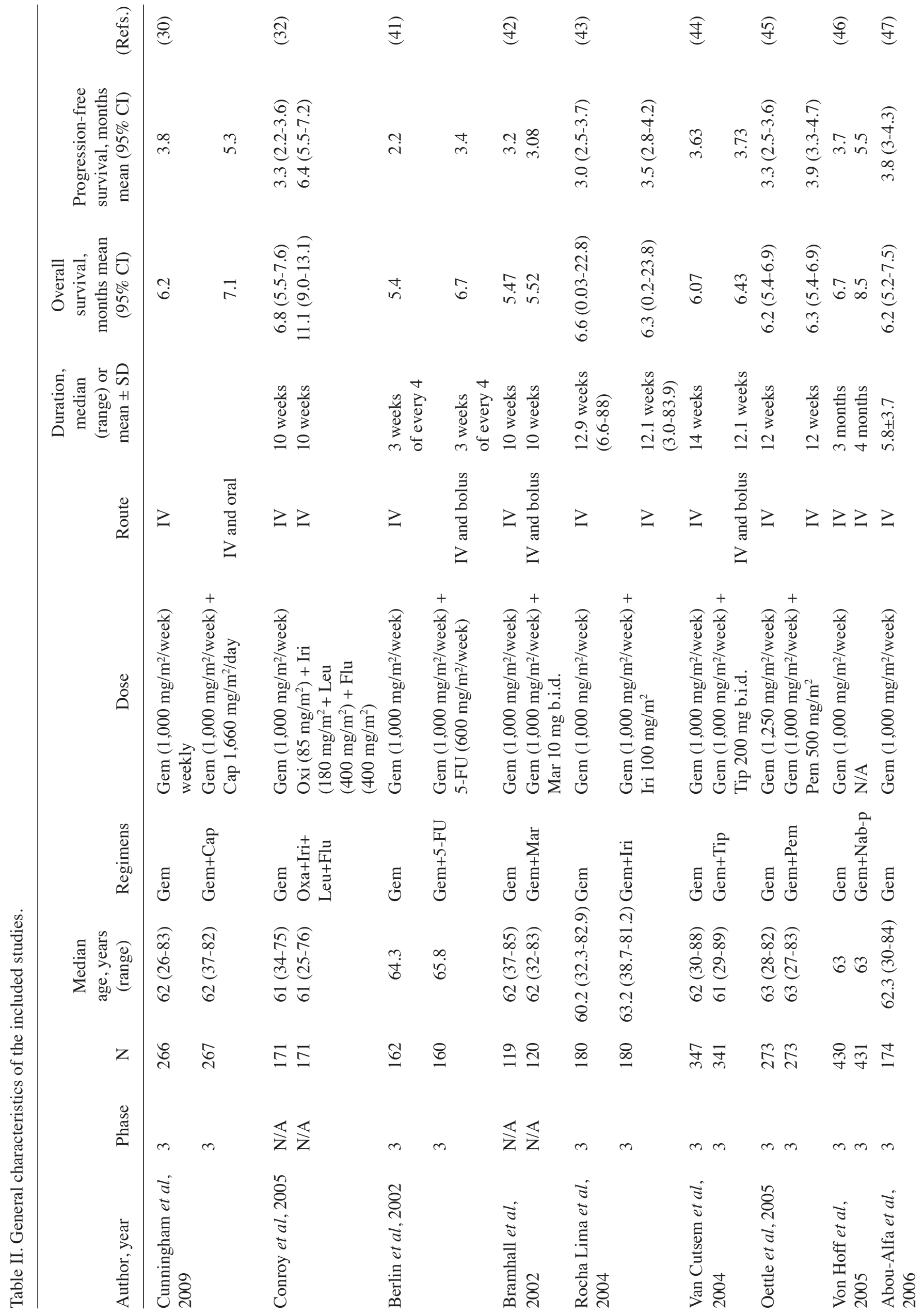




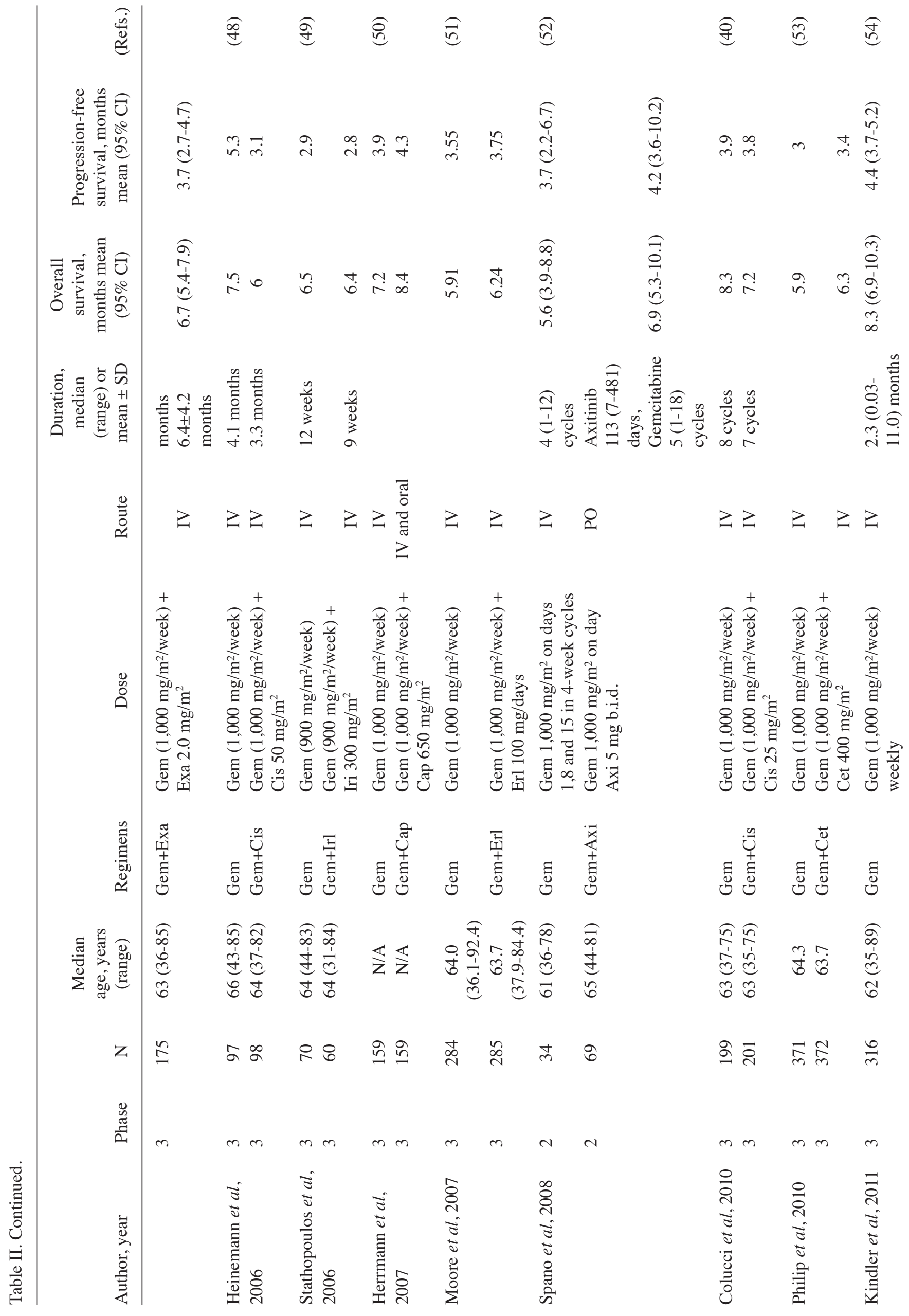




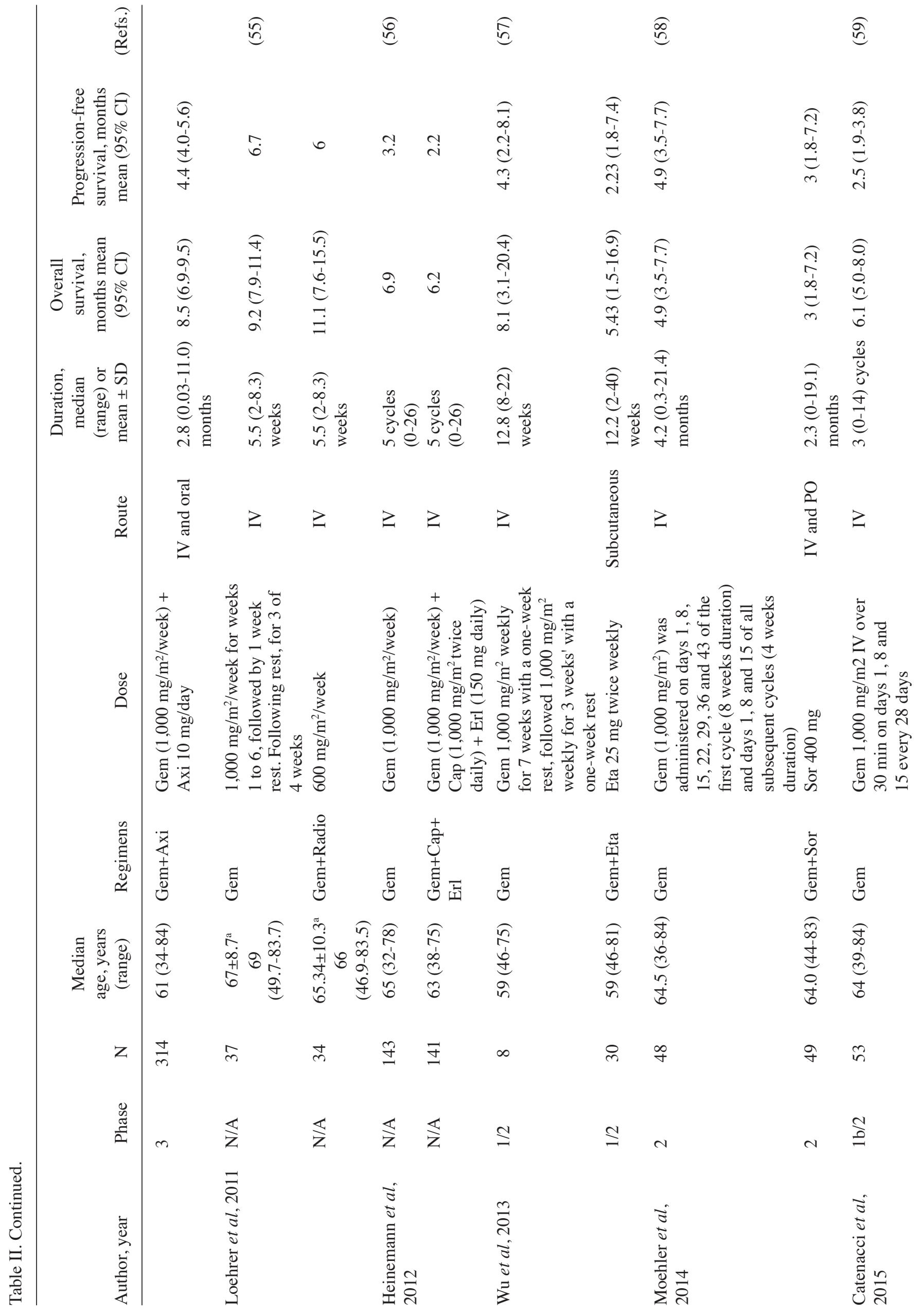




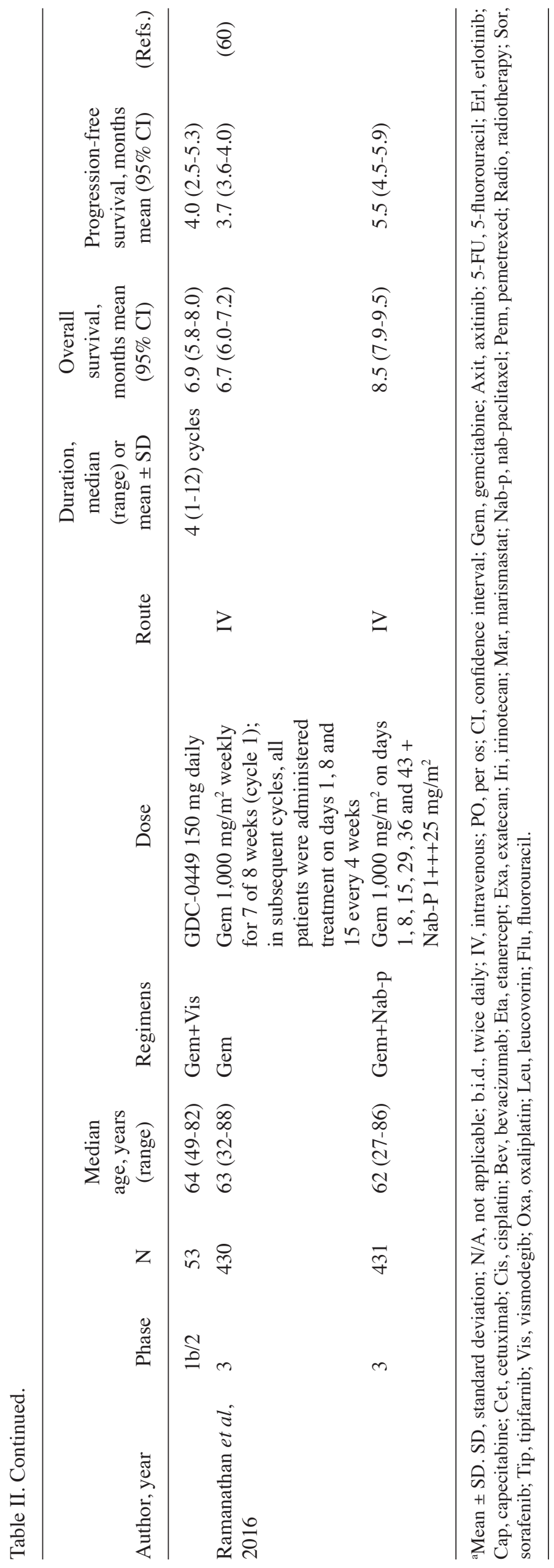




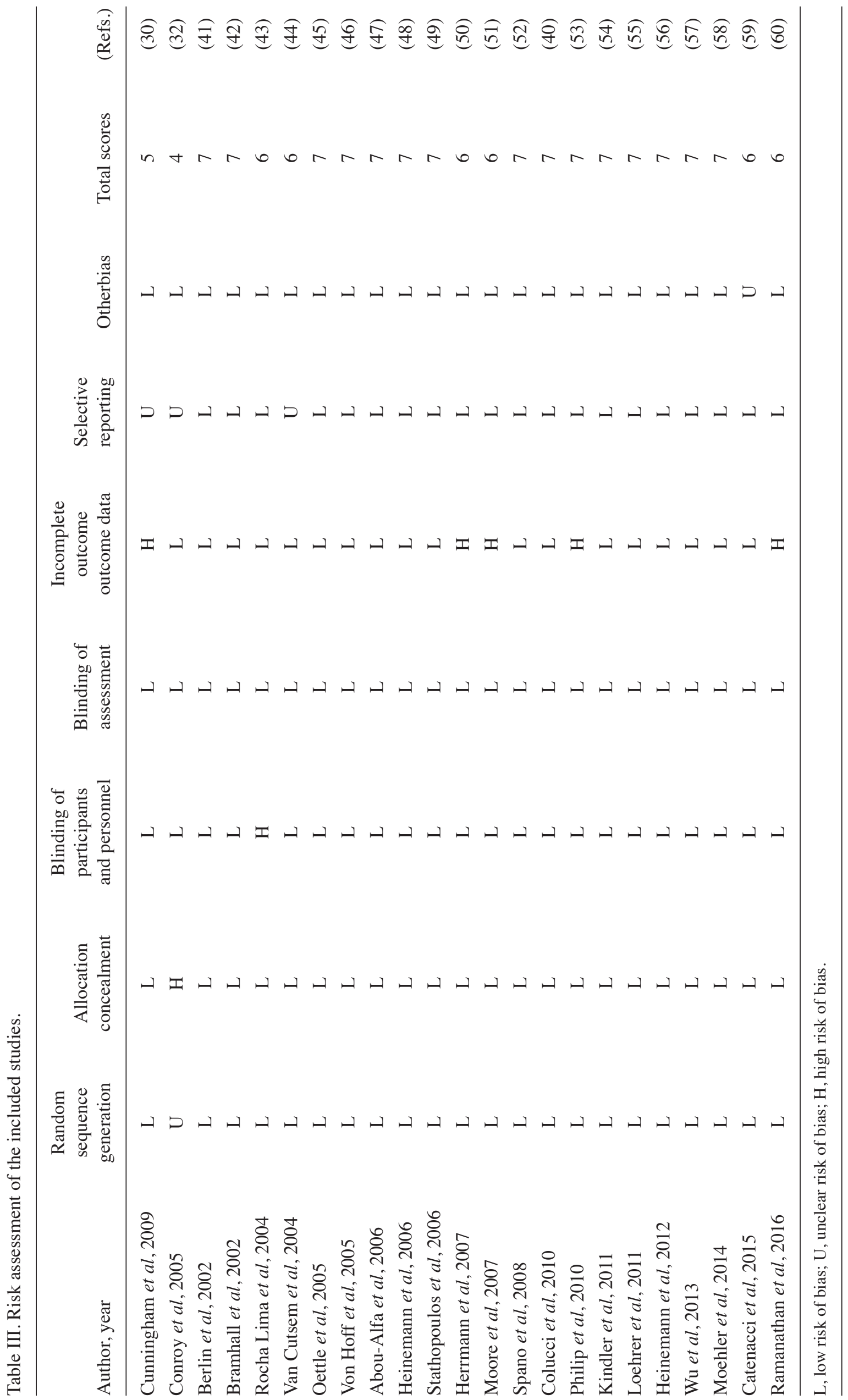


A
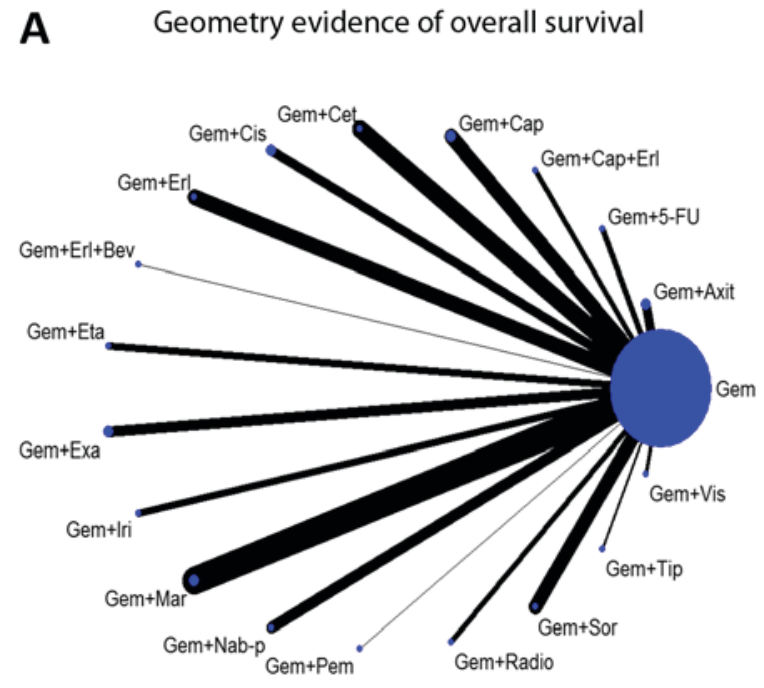

B

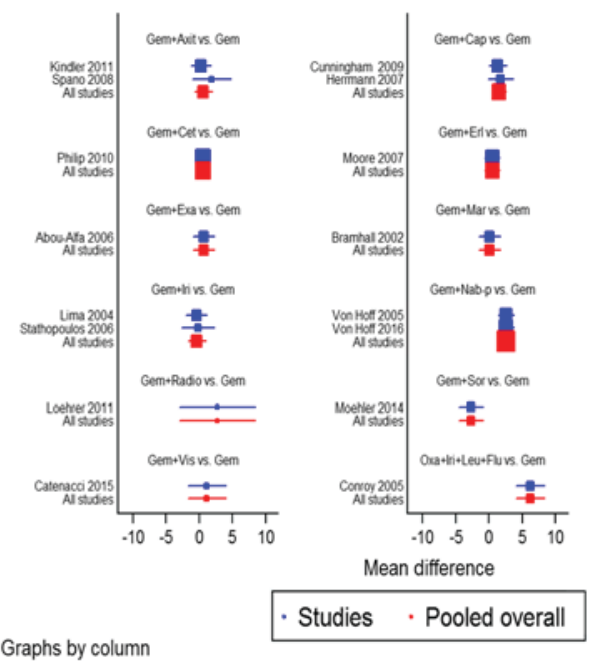

Overall survival forest

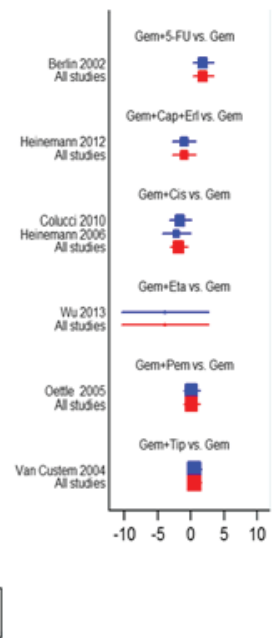

Figure 2. Network and forest plot for overall survival. (A) Geometry evidence of overall survival. (B) Overall survival forest. Gem, gemcitabine; Axit, axitinib; 5-FU, 5-fluorouracil; Erl, erlotinib; Cap, capecitabine; Cet, cetuximab; Cis, cisplatin; Bev, bevacizumab; Eta, etanercept; Exa, exatecan; Iri, irinotecan; Mar, marismastat; Nab-p, nab-paclitaxel; Pem, pemetrexed; Radio, radiotherapy; Sor, sorafenib; Tip, tipifarnib; Vis, vismodegib.

A Geometry evidence of progression-free survival

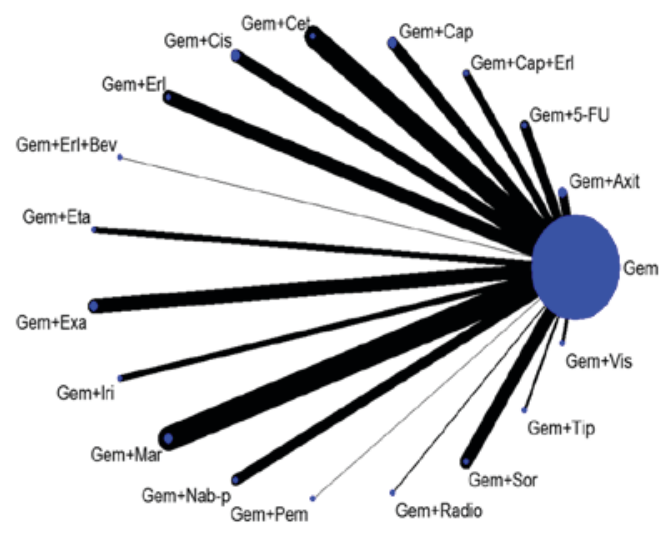

B

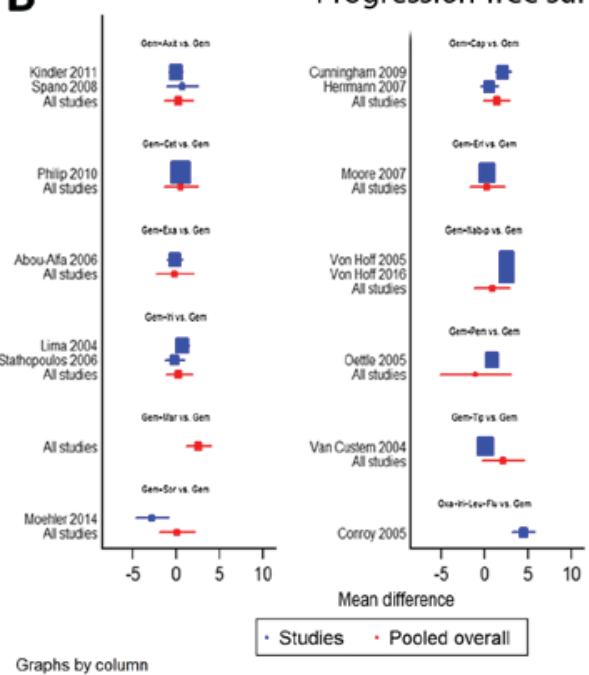

Progression-free survival forest

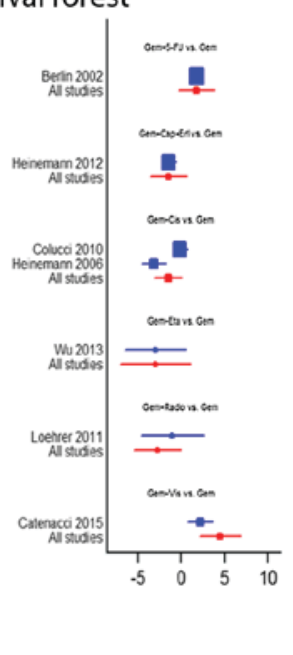

Figure 3. Network and forest plot for progression-free survival. (A) Geometry evidence of progression-free survival. (B) Progression-free survival forest. Gem, gemcitabine; Axit, axitinib; 5-FU, 5-fluorouracil; Erl, erlotinib; Cap, capecitabine; Cet, cetuximab; Cis, cisplatin; Bev, bevacizumab; Eta, etanercept; Exa, exatecan; Iri, irinotecan; Mar, marismastat; Nab-p, nab-paclitaxel; Pem, pemetrexed; Radio, radiotherapy; Sor, sorafenib; Tip, tipifarnib; Vis, vismodegib.

forest plots indicated the effectiveness of the different regimens compared with the pooled overall result (Figs. 2B and 3B).

Ranking treatments. Due to the variable conditions of APC, a critical aspect to be considered by medical doctors is what chemotherapy regimens are the most suitable and reasonable for the specific conditions of their patients. Therefore, 19 chemotherapy regimens were ranked according to their SUCRA probabilities based on OS and PFS (Fig. 4).

Regarding OS (Fig. 4A), Gem ranked 6th with a SUCRA value of 63.6. The top five combination regimens included Gem+radiotherapy (Radio), Gem+cisplatin (Cis), Gem+erlotinib (Erl)+bevacizumab (Bev), Gem+capecitabine (Cap)+Erl, and Gem+exatecan (Exa). The present results suggested that radiotherapy was the most effective treatment in extending the OS of patients, consistently with the results observed for PFS (Fig. 4B). The SUCRA scores for Gem+irinotecan (Iri) to Gem+tipifarnib presented a similar medium rank, and the scores for Gem+Cap to Gem+vismodegib (Vis) presented a low rank.

For PFS (Fig. 4B), Gem ranked 8th with a SUCRA value of 57.2. The top seven combination regimens included Gem+Radio, Gem+Erl+Bev, Gem+Cis, Gem+Cap+Erl, Gem+pemetrexed, Gem+Iri and Gem+etanercept. The SUCRA scores from Gem+ sorafenib (Sor) to Gem+ nab-paclitaxel (Nab-p) presented a medium rank, and Gem+Cap to Gem+Vis presented a low rank.

Adverse events. In addition to survival, health-associated quality of life issues are a central aspect for patients with 

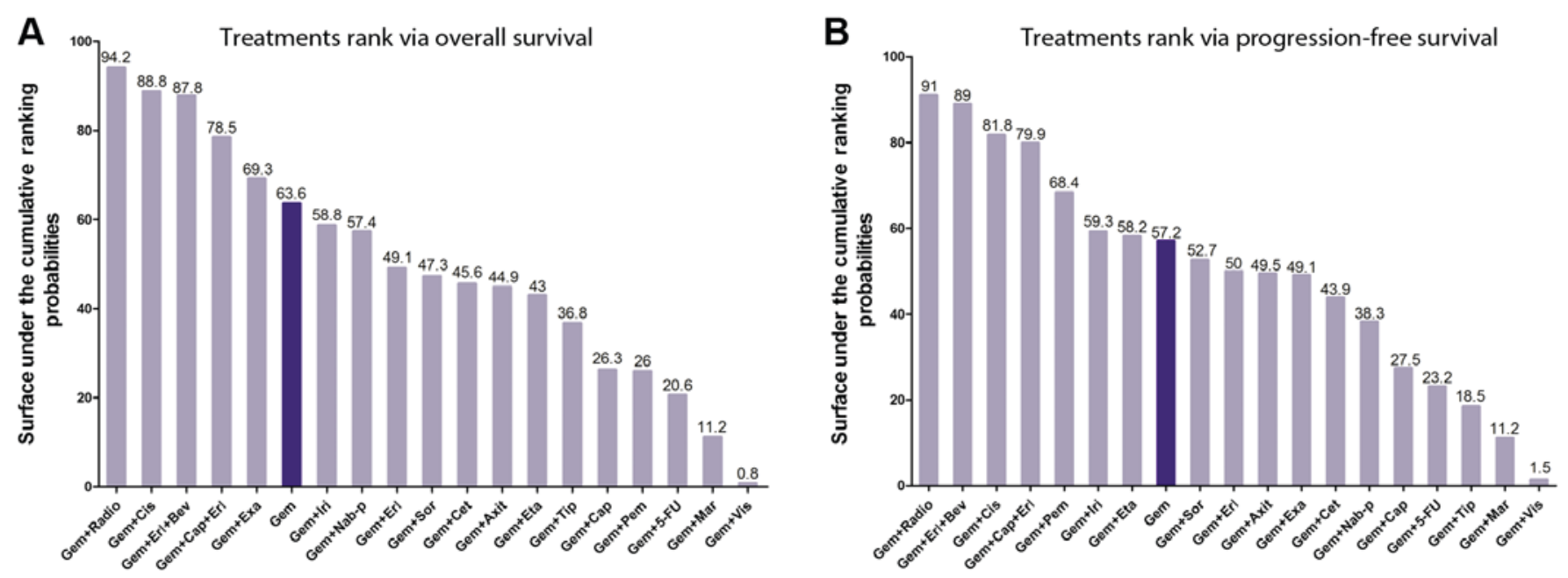

Figure 4. Treatment ranking by overall survival and progression-free survival. (A) Treatment ranking by overall survival. (B) Treatment ranking by progression-free survival. Gem, gemcitabine; Axit, axitinib; 5-FU, 5-fluorouracil; Erl, erlotinib; Cap, capecitabine; Cet, cetuximab; Cis, cisplatin; Bev, bevacizumab; Eta, etanercept; Exa, exatecan; Iri, irinotecan; Mar, marismastat; Nab-p, nab-paclitaxel; Pem, pemetrexed; Radio, radiotherapy; Sor, sorafenib; Tip, tipifarnib; Vis, vismodegib.

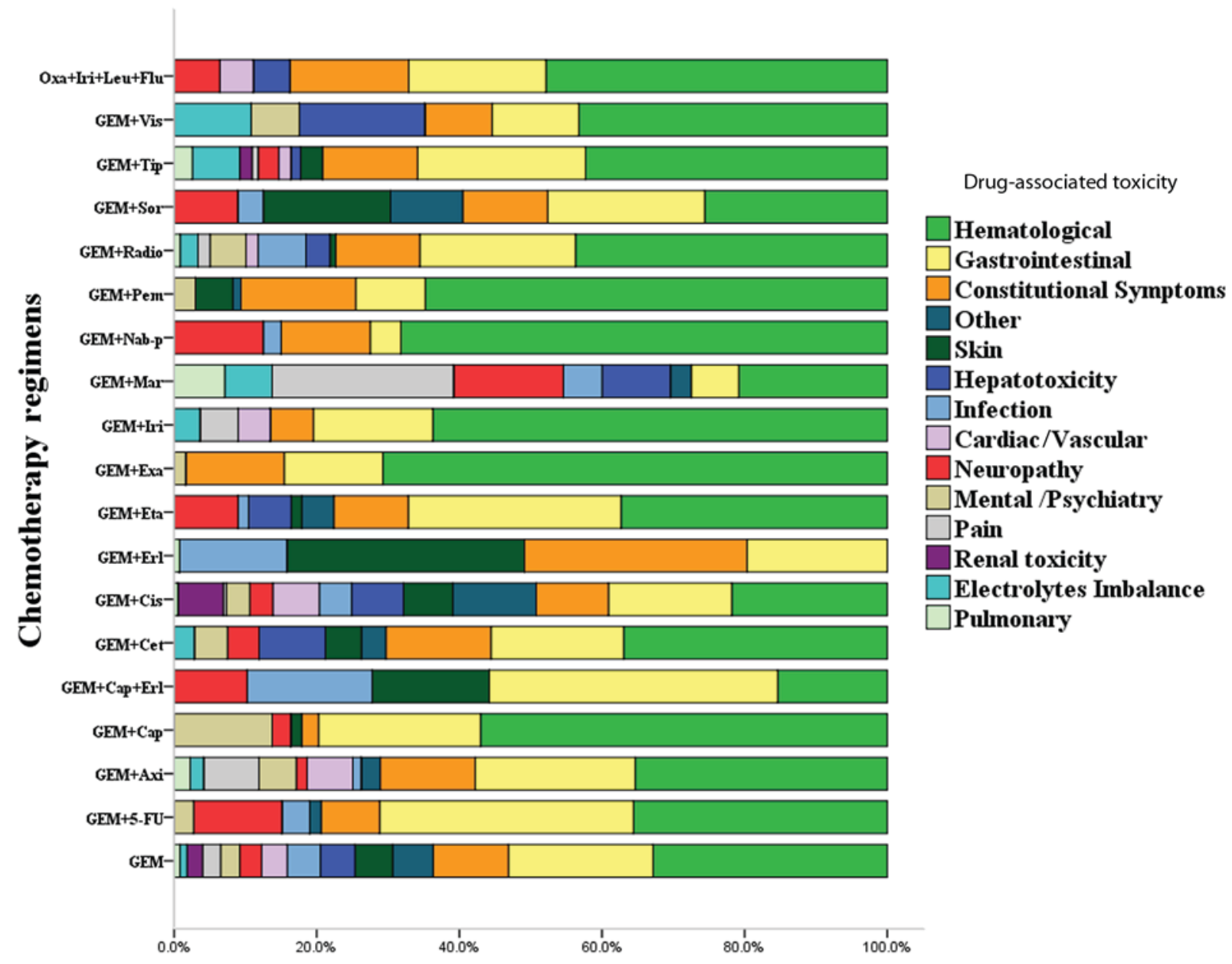

Figure 5. Assessment of the occurrence rates of 14 dominant drug-associated toxicities among 19 chemotherapy regimens. Gem, gemcitabine; Axit, axitinib; 5-FU, 5-fluorouracil; Erl, erlotinib; Cap, capecitabine; Cet, cetuximab; Cis, cisplatin; Bev, bevacizumab; Eta, etanercept; Exa, exatecan; Iri, irinotecan; Mar, marismastat; Nab-p, nab-paclitaxel; Pem, pemetrexed; Radio, radiotherapy; Sor, sorafenib; Tip, tipifarnib; Vis, vismodegib; Oxa, oxaliplatin; Leu, leucovorin; Flu, fluorouracil.

APC. Improving the health-associated quality of life by reducing treatment-associated toxicities and the occurrence rate of adverse events is important for patients. The majority of common treatment-associated toxicities include 
'hepatotoxicity', 'haematological', 'mental/psychiatry', 'renal toxicity', 'gastrointestinal', 'neuropathy', 'electrolytes imbalance', 'pain', 'infection', 'skin', 'constitutional symptoms', 'cardiac/vascular', 'pulmonary' and 'other'. The majority of these toxicities seriously affect the quality of life of the patient during the treatment process.

Regarding the 19 chemotherapy regimens, each regimen may cause various treatment-associated toxicities, and the occurrence rate of each toxicity varied among treatments. Nevertheless, the three treatment-associated toxicities with the highest occurrence rates were 'haematological', 'gastrointestinal' and 'constitutional symptoms' for all regimens except Gem+Erl, which presented the largest proportions of toxicities. Furthermore, the remaining treatment-associated toxicities, including 'skin', 'hepatotoxicity', 'infection', 'cardiac/vascular', 'neuropathy', and 'mental/psychiatry', were the most common adverse effects among the majority of regimens, following 'haematological', 'gastrointestinal' and 'constitutional symptoms'. 'Renal toxicity', 'electrolytes imbalance' and 'pulmonary' presented the lowest occurrence rate among the regimens.

Treatment-associated toxicities always accompany the therapeutic process. To achieve the best results from the perspectives of the clinicians and the patients, patients must consider a series of unavoidable treatment-associated toxicities, leading to a complex selection process.

\section{Discussion}

Since Gem was approved as a first-line treatment for APC by the FDA in 1996, a number of combination chemotherapy regimens, including $\mathrm{Gem}+\mathrm{Xs}$, have emerged. Post-treatment long-term survival remains poor and is a marked risk of the current chemotherapy regimens (24). This issue may be caused by a failure of local control and of diagnosing localized APC in time (25). Associated RCTs and studies published between 2002 and 2016, covering a total of 14 years, were selected to assess the advantages and disadvantages of each regimen compared with Gem monotherapy.

Among the chemotherapy regimens, Gem+Radio presented the principal improvement in extending OS and PFS. This finding suggested that radiotherapy may block the progressive deterioration associated with advanced cancer and is consistent with the previous study of Youl et al (26), which identified that a gross tumor volume $<48 \mathrm{~cm}^{3}$ may be successfully targeted with radiotherapy. An additional three regimens, Gem+Cis, Gem+Erl+Bev and Gem+Cap+Erl, were better compared with Gem monotherapy in terms of OS and PFS. Regarding other combination regimens, Gem+Iri, Gem+Sor, Gem+Erl, Gem+Axitinib, Gem+Exa, Gem+Cetuximab, Gem+Nab-p and Gem+Cap presented higher SUCRA probabilities compared with Gem+5-FU, another double regimen. In contrast, Gem+marismastat and Gem+Vis exhibited decreased SUCRA probabilities. Although numerous regimens are available, side effects always accompany the therapeutic effect. Therefore, selecting the regimen that may offer the longest survival is a complex process that requires the clinician to comprehensively assess the disease status of the patient in order to identify a balance between the benefits and risks of the treatment.

A previous study demonstrated that patients who undergo 5-FU and 5-FU-based regimens following resection had improved survival rates (27). 5-FU has been used as the principle chemoradiation and/or chemotherapy regimens and was previously considered the principal effective chemotherapeutic agent available in pancreatic cancer treatment. In the 1990s, Gem was first demonstrated to be a safe drug with low toxicity for APC treatment (28). Subsequent clinical trials demonstrated the significant advantages of Gem for short-term and long-term OS in the treatment of advanced and metastatic pancreatic cancer $(8,29,30)$; these previous results demonstrated the effectiveness of Gem therapy in pancreatic cancer.

In patients with optimal performance status, concurrent chemotherapy, including FOLFIRINOX, albumin-bound paclitaxel and 5-FU, in combination with Gem, or other Gem-based combined chemotherapies, may provide increased survival benefits $(31,32)$. Although patients at similar disease-stages receive the same chemical regimens, there may be various outcomes due to individual variations.

Gem has been the standard for treating APC since 1996, providing a limited survival of 6 months due to the intrinsic capacity of cancer cells and the surrounding microenvironment to resist cytotoxicity $(33,34)$. Combination therapies with Gem have presented limited effectiveness in clinical trials, and the identification of novel therapeutic strategies that may increase median survival and PFS with reduced adverse effects, is required. Certain treatments, including 5-FU monotherapy, presented significantly decreased survival compared with Gem monotherapy and have demonstrated the effectiveness of Gem as a first-line chemotherapy for APC. Although certain trials examining combination therapies demonstrated improved objective response rates, the treatments failed to achieved improvement in all three of the most common outcomes measured; Median survival, PFS and the objective response rate (35-39). The present network meta-analysis of patients with unresectable pancreatic cancer suggested that the current median survival for patients treated with Gem was $>6$ months, and the objective response rate for Gem ranged between 4.4 and $17.3 \%$ (40). These results may represent the standard to be compared with future results of single-arm phase II trials involving Gem-based combination regimens. The severity of the adverse effects among these regimens was assessed by inconsistent scales, which may contribute to bias. Future studies may consider a consistent scale among various conditions in order to avoid possible biases.

In conclusion, numerous chemotherapy regimens are used to extend the survival and reduce the treatment-associated toxicities of patients with APC. The effect and treatment-associated toxicities of a particular regimen requires consideration to balance the benefits and risks for the patient. The present study provides additional evidence for selecting appropriate treatments according to the clinical situation.

\section{Acknowledgements}

The authors would like to thank Dr Kun Huang from Chengdu TME Co., Ltd. (Chengdu, China) for advice on optimizing the data search strategy and Professor Jinhui Tian from Lanzhou University (Lanzhou, China) for the valuable discussion regarding the implications of the results. 


\section{Funding}

The present study was supported by the Science \& Technology Department of Sichuan Province Grants (Sichuan, China; grant no. 2016JY0036).

\section{Availability of data and materials}

The analyzed data sets generated during the study are available from the corresponding author on reasonable request.

\section{Authors' contributions}

JC, FX and SY conceived and designed the present study. YX, LC, JY, XW, ZZ and NL performed the data extraction. JY and FX ensured the quality of the data. FX and SY analyzed the data. JC, FX and LC drafted the manuscript. All authors read and approved the final manuscript.

\section{Ethics approval and consent to participate}

Not applicable.

\section{Patient consent for publication}

Not applicable.

\section{Competing interests}

The authors declare that they have no competing interests.

\section{References}

1. Goral V: Pancreatic cancer: Pathogenesis and diagnosis. Asian Pac J Cancer Prev 16: 5619-5624, 2015.

2. Pancreatic Cancer Action Network: The alarming rise of pancreatic cancer deaths in the United States: Why we need to stem the tide today. Pancreatic Cancer Action Network, Manhattan Beach, CA, 2012.

3. Krechler T, Horejs J, Ulrych J, Zeman M, Macásek J, Dusková J and Zák A: Current status of pancreatic cancer diagnosis. Cas Lek Cesk 150: 587-593, 2011 (In Czech).

4. Freelove R and Walling AD: Pancreatic cancer: Diagnosis and management. Am Fam Physician 73: 485-492, 2006.

5. Chen WQ, Liang D, Zhang SW, Zheng RS and He YT: Pancreatic cancer incidence and mortality patterns in china, 2009. Asian Pac J Cancer Prev 14: 7321-7324, 2013.

6. Wang Y, Schrag D, Brooks GA and Dominici F: National trends in pancreatic cancer outcomes and pattern of care among Medicare beneficiaries, 2000 through 2010. Cancer 120: 1050-1058, 2014.

7. Akhtar-Danesh GG, Finley C and Akhtar-Danesh N: Long-term trends in the incidence and relative survival of pancreatic cancer in Canada: A population-based study. Pancreatology 16: 259-265, 2016.

8. Burris HA III, Moore MJ, Andersen J, Green MR, Rothenberg ML, Modiano MR, Cripps MC, Portenoy RK, Storniolo AM, Tarassoff P, et al: Improvements in survival and clinical benefit with gemcitabine as first-line therapy for patients with advanced pancreas cancer: A randomized trial. J Clin Oncol 15: 2403-2413, 1997.

9. Abbruzzese JL: New applications of gemcitabine and future directions in the management of pancreatic cancer. Cancer 95(Suppl 4): 941-945, 2002.

10. Macdonald JS: Clinical overview: Adjuvant therapy of gastrointestinal cancer. Cancer Chemother Pharmacol 54 (Suppl 1): S4-S11, 2004.

11. Okusaka T, Ishii H, Funakoshi A, Ueno H, Furuse J and Sumii T: A phase I/II study of combination chemotherapy with gemcitabine and 5-fluorouracil for advanced pancreatic cancer. Jpn J Clin Oncol 36: 557-563, 2006.
12. Shi S, Yao W, Xu J, Long J, Liu C and Yu X: Combinational therapy: New hope for pancreatic cancer? Cancer Lett 317: 127-135, 2012.

13. Kristensen A, Vagnildhaug OM, Grønberg BH, Kaasa S, Laird B and Solheim TS: Does chemotherapy improve health-related quality of life in advanced pancreatic cancer? A systematic review. Crit Rev Oncol Hematol 99: 286-298, 2016.

14. Gresham GK, Wells GA, Gill S, Cameron C and Jonker DJ: Chemotherapy regimens for advanced pancreatic cancer: A systematic review and network meta-analysis. BMC Cancer 14: 471, 2014.

15. Ansari D, Tingstedt B, Andersson B, Holmquist F, Sturesson C, Williamsson C, Sasor A, Borg D, Bauden M and Andersson R: Pancreatic cancer: Yesterday, today and tomorrow. Future Oncol 12: 1929-1946, 2016.

16. Siegel RL, Miller KD and Jemal A: Cancer statistics, 2016. CA Cancer J Clin 66: 7-30, 2016.

17. Birhanu G, Javar HA, Seyedjafari E and Zandi-Karimi A: Nanotechnology for delivery of gemcitabine to treat pancreatic cancer. Biomed Pharmacother 88: 635-643, 2017.

18. Moher D, Liberati A, Tetzlaff J and Altman DG; PRISMA Group: Preferred reporting items for systematic reviews and meta-analyses: The PRISMA statement. PLoS Med 6: e1000097, 2009.

19. Chaimani A, Higgins JP, Mavridis D, Spyridonos P and Salanti G: Graphical tools for network meta-analysis in STATA. PLoS One 8: e76654, 2013.

20. Biermann JS, Chow W, Reed DR, Lucas D, Adkins DR, Agulnik M, Benjamin RS, Brigman B, Budd GT, Curry WT, et al: NCCN guidelines insights: Bone cancer, version 2.2017. J Natl Compr Canc Netw 15: 155-167, 2017.

21. Hernández-Quiles C, Bernabeu-Wittel M, Pérez-Belmonte LM, Macías-Mir P, Camacho-González D, Massa B, Maiz-Jiménez M and Ollero-Baturone M; PALIAR investigators: Concordance of Barthel Index, ECOG-PS, and Palliative Performance Scale in the assessment of functional status in patients with advanced medical diseases. BMJ Support Palliat Care 7: 300-307, 2017.

22. Lovato N, Lack L, Wright H and Kennaway DJ: Evaluation of a brief treatment program of cognitive behavior therapy for insomnia in older adults. Sleep 37: 117-126, 2014.

23. Salanti G, Ades AE and Ioannidis JP: Graphical methods and numerical summaries for presenting results from multiple-treatment meta-analysis: An overview and tutorial. J Clin Epidemiol 64: 163-171, 2011.

24. Jutric $\mathrm{Z}$ and Melstrom LG: New treatment options and management considerations in borderline resectable pancreatic cancer. Oncology (Williston Park) 31: 443-452, 2017.

25. Sugarbaker PH: Strategies to improve local control of resected pancreas adenocarcinoma. Surg Oncol 26: 63-70, 2017.

26. Youl M, Hashem S, Brade A, Cummings B, Dawson LA, Gallinger S, Hedley D, Jiang H, Kim J, Krzyzanowska MK, et al: Induction gemcitabine plus concurrent gemcitabine and radiotherapy for locally advanced unresectable or resected pancreatic cancer. Clin Oncol (R Coll Radiol) 26: 203-209, 2014.

27. Kalser $\mathrm{MH}$ and Ellenberg SS: Pancreatic cancer. Adjuvant combined radiation and chemotherapy following curative resection. Arch Surg 120: 899-903, 1985.

28. Aapro MS, Martin C and Hatty S: Gemcitabine-a safety review. Anticancer Drugs 9: 191-201, 1998.

29. Regine WF, Winter KA, Abrams RA, Safran H, Hoffman JP, Konski A, Benson AB, Macdonald JS, Kudrimoti MR, Fromm ML, et al: Fluorouracil vs gemcitabine chemotherapy before and after fluorouracil-based chemoradiation following resection of pancreatic adenocarcinoma: A randomized controlled trial. JAMA 299: 1019-1026, 2008.

30. Cunningham D, Chau I, Stocken DD, Valle JW, Smith D, Steward W, Harper PG, Dunn J, Tudur-Smith C, West J, et al: Phase III randomized comparison of gemcitabine versus gemcitabine plus capecitabine in patients with advanced pancreatic cancer. J Clin Oncol 27: 5513-5518, 2009.

31. Vaccaro V, Sperduti I and Milella M: FOLFIRINOX versus gemcitabine for metastatic pancreatic cancer. N Engl J Med 365: 768-769; author reply 769, 2011.

32. Conroy T, Desseigne F, Ychou M, Bouché O, Guimbaud R, Bécouarn Y, Adenis A, Raoul JL, Gourgou-Bourgade S, de la Fouchardière $\mathrm{C}$, et al: FOLFIRINOX versus gemcitabine for metastatic pancreatic cancer. N Engl J Med 364: 1817-1825, 2011.

33. Rhim AD, Oberstein PE, Thomas DH, Mirek ET, Palermo CF, Sastra SA, Dekleva EN, Saunders T, Becerra CP, Tattersall IW, et al: Stromal elements act to restrain, rather than support, pancreatic ductal adenocarcinoma. Cancer Cell 25: 735-747, 2014. 
34. Fox RG, Lytle NK, Jaquish DV, Park FD, Ito T, Bajaj J, Koechlein CS, Zimdahl B, Yano M, Kopp J, et al: Image-based detection and targeting of therapy resistance in pancreatic adenocarcinoma. Nature 534: 407-411, 2016.

35. Citterio C, Baccini M, Orlandi E, Di Nunzio C and Cavanna L: Second-line chemotherapy for the treatment of metastatic pancreatic cancer after first-line gemcitabine-based chemotherapy: A network meta-analysis. Oncotarget 9: 29801-29809, 2018.

36. Chiorean EG, Von Hoff DD, Tabernero J, El-Maraghi R, Wee Ma W, Reni M, Harris M, Whorf R, Liu H, Shiansong Li J, et al: Second-line therapy after nab-paclitaxel plus gemcitabine or after gemcitabine for patients with metastatic pancreatic cancer. Br J Cancer 115: e13, 2016.

37. Oettle H, Riess H, Stieler JM, Heil G, Schwaner I, Seraphin J, Görner M, Mölle M, Greten TF, Lakner V, et al: Second-line oxaliplatin, folinic acid, and fluorouracil versus folinic acid and fluorouracil alone for gemcitabine-refractory pancreatic cancer: Outcomes from the CONKO-003 trial. J Clin Oncol 32: 2423-2429, 2014

38. Walker EJ and Ko AH: Beyond first-line chemotherapy for advanced pancreatic cancer: An expanding array of therapeutic options? World J Gastroenterol 20: 2224-2236, 2014.

39. Schultheis B, Reuter D, Ebert MP, Siveke J, Kerkhoff A, Berdel WE, Hofheinz R, Behringer DM, Schmidt WE, Goker E, et al: Gemcitabine combined with the monoclonal antibody nimotuzumab is an active first-line regimen in KRAS wildtype patients with locally advanced or metastatic pancreatic cancer: A multicenter, randomized phase IIb study. Ann Oncol 28: 2429-2435, 2017

40. Colucci G, Giuliani F, Gebbia V, Biglietto M, Rabitti P, Uomo G, Cigolari S, Testa A, Maiello E and Lopez M: Gemcitabine alone or with cisplatin for the treatment of patients with locally advanced and/or metastatic pancreatic carcinoma: A prospective, randomized phase III study of the Gruppo Oncologia dell'Italia Meridionale. Cancer 94: 902-910, 2002

41. Berlin JD, Catalano P, Thomas JP, Kugler JW, Haller DG and Benson AB III: Phase III study of gemcitabine in combination with fluorouracil versus gemcitabine alone in patients with advanced pancreatic carcinoma: Eastern Cooperative Oncology Group Trial E2297. J Clin Oncol 20: 3270-3275, 2002.

42. Bramhall SR, Schulz J, Nemunaitis J, Brown PD, Baillet M and Buckels JA: A double-blind placebo-controlled, randomised study comparing gemcitabine and marimastat with gemcitabine and placebo as first line therapy in patients with advanced pancreatic cancer. Br J Cancer 87: 161-167, 2002

43. Rocha Lima CM, Green MR, Rotche R, Miller WH Jr, Jeffrey GM, Cisar LA, Morganti A, Orlando N, Gruia G and Miller LL: Irinotecan plus gemcitabine results in no survival advantage compared with gemcitabine monotherapy in patients with locally advanced or metastatic pancreatic cancer despite increased tumor response rate. J Clin Oncol 22: 3776-3783, 2004

44. Van Cutsem E, van de Velde H, Karasek P, Oettle H, Vervenne WL, Szawlowski A, Schoffski P, Post S, Verslype C, Neumann $\mathrm{H}$, et al: Phase III trial of gemcitabine plus tipifarnib compared with gemcitabine plus placebo in advanced pancreatic cancer. J Clin Oncol 22: 1430-1438, 2004

45. Oettle H, Richards D, Ramanathan RK, van Laethem JL, Peeters M, Fuchs M, Zimmermann A, John W, Von Hoff D, Arning $M$ and Kindler HL: A phase III trial of pemetrexed plus gemcitabine versus gemcitabine in patients with unresectable or metastatic pancreatic cancer. Ann Oncol 16: 1639-1645, 2005.

46. Von Hoff DD, Ervin TJ, Arena FP, Gabriela Chiorean E, Infante JR, Moore M, Seay TE, Tjulandin S, Ma WW, Saleh M, et al: Randomized phase III study of weekly nabpaclitaxel plus gemcitabine versus gemcitabine alone in patients with metastatic adenocarcinoma of the pancreas (MPACT). J Clin Oncol 31: LBA148-LBA148, 2013.

47. Abou-Alfa GK, Letourneau R, Harker G, Modiano M, Hurwitz H, Tchekmedyian NS, Feit K, Ackerman J, De Jager RL, Eckhardt SG and O'Reilly EM: Randomized phase III study of exatecan and gemcitabine compared with gemcitabine alone in untreated advanced pancreatic cancer. J Clin Oncol 24: 4441-4447, 2006.

48. Heinemann V, Quietzsch D, Gieseler F, Gonnermann M, Schönekäs H, Rost A, Neuhaus H, Haag C, Clemens M, Heinrich B, et al: Randomized phase III trial of gemcitabine plus cisplatin compared with gemcitabine alone in advanced pancreatic cancer. J Clin Oncol 24: 3946-3952, 2006.
49. Stathopoulos GP, Syrigos K, Aravantinos G, Polyzos A, Papakotoulas P, Fountzilas G, Potamianou A, Ziras N, Boukovinas J, Varthalitis J, et al: A multicenter phase III trial comparing irinotecan-gemcitabine (IG) with gemcitabine (G) monotherapy as first-line treatment in patients with locally advanced or metastatic pancreatic cancer. Br J Cancer 95: 587-592, 2006.

50. Herrmann R, Bodoky G, Ruhstaller T, Glimelius B, Bajetta E, Schüller J, Saletti P, Bauer J, Figer A, Pestalozzi B, et al: Gemcitabine plus capecitabine compared with gemcitabine alone in advanced pancreatic cancer: A randomized, multicenter, phase III trial of the Swiss Group for Clinical Cancer Research and the Central European Cooperative Oncology Group. J Clin Oncol 25: 2212-2217, 2007

51. Moore MJ, Goldstein D, Hamm J, Figer A, Hecht JR, Gallinger S, Au HJ, Murawa P, Walde D, Wolff RA, et al: Erlotinib plus gemcitabine compared with gemcitabine alone in patients with advanced pancreatic cancer: A phase III trial of the National Cancer Institute of Canada Clinical Trials Group. J Clin Oncol 25: 1960-1966, 2007.

52. Spano JP, Chodkiewicz C, Maurel J, Wong R, Wasan H, Barone C, Létourneau R, Bajetta E, Pithavala Y, Bycott P, et al: Efficacy of gemcitabine plus axitinib compared with gemcitabine alone in patients with advanced pancreatic cancer: An open-label randomised phase II study. Lancet 371: 2101-2108, 2008.

53. Philip PA, Benedetti J, Corless CL, Wong R, O'Reilly EM, Flynn PJ, Rowland KM, Atkins JN, Mirtsching BC, Rivkin SE, et al: Phase III study comparing gemcitabine plus cetuximab versus gemcitabine in patients with advanced pancreatic adenocarcinoma: Southwest Oncology Group-directed intergroup trial S0205. J Clin Oncol 28: 3605-3610, 2010.

54. Kindler HL, Ioka T, Richel DJ, Bennouna J, Létourneau R, Okusaka T, Funakoshi A, Furuse J, Park YS, Ohkawa S, et al: Axitinib plus gemcitabine versus placebo plus gemcitabine in patients with advanced pancreatic adenocarcinoma: A double-blind randomised phase 3 study. Lancet Oncol 12: 256-262, 2011.

55. Loehrer PJ Sr, Feng Y, Cardenes H, Wagner L, Brell JM, Cella D, Flynn P, Ramanathan RK, Crane CH, Alberts SR and Benson AB III: Gemcitabine alone versus gemcitabine plus radiotherapy in patients with locally advanced pancreatic cancer: An Eastern Cooperative Oncology Group trial. J Clin Oncol 29: 4105-4112, 2011.

56. Heinemann V, Vehling-Kaiser U, Waldschmidt D, Kettner E, Märten A, Winkelmann C, Klein S, Kojouharoff G, Gauler TC, von Weikersthal LF, et al: Gemcitabine plus erlotinib followed by capecitabine versus capecitabine plus erlotinib followed by gemcitabine in advanced pancreatic cancer: Final results of a randomised phase 3 trial of the 'Arbeitsgemeinschaft Internistische Onkologie' (AIO-PK0104). Gut 62: 751-759, 2013.

57. Wu C, Fernandez SA, Criswell T, Chidiac TA, Guttridge D, Villalona-Calero M and Bekaii-Saab TS: Disrupting cytokine signaling in pancreatic cancer: A phase I/II study of etanercept in combination with gemcitabine in patients with advanced disease. Pancreas 42: 813-818, 2013

58. Moehler M, Maderer A, Schimanski C, Kanzler S, Denzer U, Kolligs FT, Ebert MP, Distelrath A, Geissler M, Trojan J, et al: Gemcitabine plus sorafenib versus gemcitabine alone in advanced biliary tract cancer: A double-blind placebo-controlled multicentre phase II AIO study with biomarker and serum programme. Eur J Cancer 50: 3125-3135, 2014.

59. Catenacci DV, Junttila MR, Karrison T, Bahary N, Horiba MN, Nattam SR, Marsh R, Wallace J, Kozloff M, Rajdev L, et al: Randomized phase Ib/II study of gemcitabine plus placebo or vismodegib, a hedgehog pathway inhibitor, in patients with metastatic pancreatic cancer. J Clin Oncol 33: 4284-4292, 2015.

60. Ramanathan RK, Goldstein D, Korn RL, Arena F, Moore M, Siena S, Teixeira L, Tabernero J, Van Laethem JL, Liu H, et al: Positron emission tomography response evaluation from a randomized phase III trial of weekly nab-paclitaxel plus gemcitabine versus gemcitabine alone for patients with metastatic adenocarcinoma of the pancreas. Ann Oncol 27: 648-653, 2016.

This work is licensed under a Creative Commons Attribution 4.0 International (CC BY 4.0) License. 